\title{
ULTRA-HIGH RESOLUTION ATOMIC AND MOLECULAR SPECTROSCOPY AT THE ADVANCED LIGHT SOURCE*
}

\author{
F. SChlachter AND J. BozeK \\ Advanced Light Source, Lawrence Berkeley National Laboratory \\ Berkeley CA 94720 , USA
}

\begin{abstract}
Ultra-bright ultraviolet and soft X-ray beams produced at the Advanced Light Source are achieving world records in spectroscopic resolution. High-resolution spectroscopy in the 20 to $300 \mathrm{eV}$ photon energy range has been applied to photoionization yield and photoemission measurements in atoms and molecules to reveal previously unseen resonance series in doubly-excited autoionizing states of helium, to analyze angular distributions in photoemission from doubly-excited states of helium and other rare gases, to reveal vibrational and ligand-field splitting of small molecules through photoionization, and, to examine photoemission from doubly- and triply-excited hollow lithium atomic states.
\end{abstract}

PACS numbers: $32.80 .-\mathrm{t}$

\section{Introduction}

Third-generation light sources are characterized by the ultra-high-brightness photon beams they produce. This is due to the low emittance and small size of the electron or positron beams used to produce the radiation, and the use of undulators. High brightness allows a beam of photons to be focused to a small spot, a capability that is highly valued in many kinds of microscopy, with potential applications in technological and materials sciences. High brightness also leads to the production of a partially coherent beam of photons, which is useful for both interferometry and specialized microscopes. Finally, high brightness allows high spectral resolution with high flux because the entire photon beam from an undulator can be focused to pass through the entrance slit of a monochromator. This high spectral resolution allows spectroscopy of atoms and molecules with unprecedented resolution.

The Advanced Light Source (ALS) in Berkeley was the first low-energy (1.0-1.9 GeV) third-generation light source to produce high brightness photon

*This work was supported by the Director, Office of Energy Research, Office of Basic Energy Sciences, Materials Sciences Division of the U.S. Department of Energy, under Contract No. DE-AC03-76SF00098. 
beams in the vacuum ultraviolet (VUV) and soft X-ray region of the spectrum. The ALS has since been joined by other low-energy light sources, including SRRC in Taiwan, PLS in Korea, and ELETTRA in Trieste. The first high-energy (6 GeV) third-generation light source was ESRF in Grenoble, which uses positrons to produce high brightness X-rays. ESRF has recently been joined in the high-energy range by APS at Argonne (USA), and will be joined in a few years by SPring- 8 in Japan. High- and low-energy light sources provide complementary coverage of the spectral range from infrared through hard X-rays.

Photons are an excellent probe of atoms and molecules. At energies too low for Compton scattering, a photon which interacts with an atom gives all its energy to one electron, or to several electrons through electron-electron correlation (Coulomb repulsion) or through the interaction of electrons in the atom. The hydrogen atom contains only one electron, the only force is central (along the axis between the nucleus and the one electron), and the equation of motion can be solved exactly. In contrast, the helium atom with two electrons includes electron-electron correlation where one of the forces between the two electrons is not central. In this case, the equations of motion of the three particles cannot be solved exactly; quantum-mechanical interference produces an exquisitely complicated spectrum.

Many techniques are used to study photoionization of atoms and molecules. We shall show results using two methods: photoionization in a gas cell, and electron spectroscopy using an electrostatic energy analyzer. Photoionization yield measures the total cross section for all processes because electrons or ions in a gas cell are collected onto electrodes by a transverse electric field. Photoionization yield measurements have the advantage of very high sensitivity, because not only can high target densities be used, but the collection efficiency for reaction products is $100 \%$. However, because photoionization yield measures integrated cross sections, individual physical processes cannot be distinguished. Photoionization with electron-energy analysis allows different physical processes to be distinguished on the basis of electron energy and angular distribution. The use of low-density targets, a small solid angle for electron collection, and scanning the electron energy spectrum, require that relatively long measurement times should be used. The two methods are thus complementary.

The measurements described in this paper were performed on Beamline 9.0.1 at the ALS [1]. This beam line consists of a spherical-grating monochromator with three gratings and a moveable exit slit, and the radiation source is a $4.5-\mathrm{m}$ undulator with a period of $8 \mathrm{~cm}(10 \mathrm{~cm}$ after 1995). The design resolving power $(E / \Delta E)$ of the monochromator is 10000 , and the design flux at this resolving power is greater than $10^{12}$ photons/second in the energy range $20-300 \mathrm{eV}$. Under certain circumstances the resolving power can be as high as $64000(1 \mathrm{meV}$ at $64 \mathrm{eV}$ ), albeit at significantly reduced flux [2]. This resolving power is a factor of 4 better than has ever been achieved in the soft X-ray region. 


\section{Helium}

Understanding the three-body Coulomb system is central to atomic physics, and the helium atom is the prototypical and most simple two-electron three-body system. Electron-electron correlation is fundamental to the understanding of the
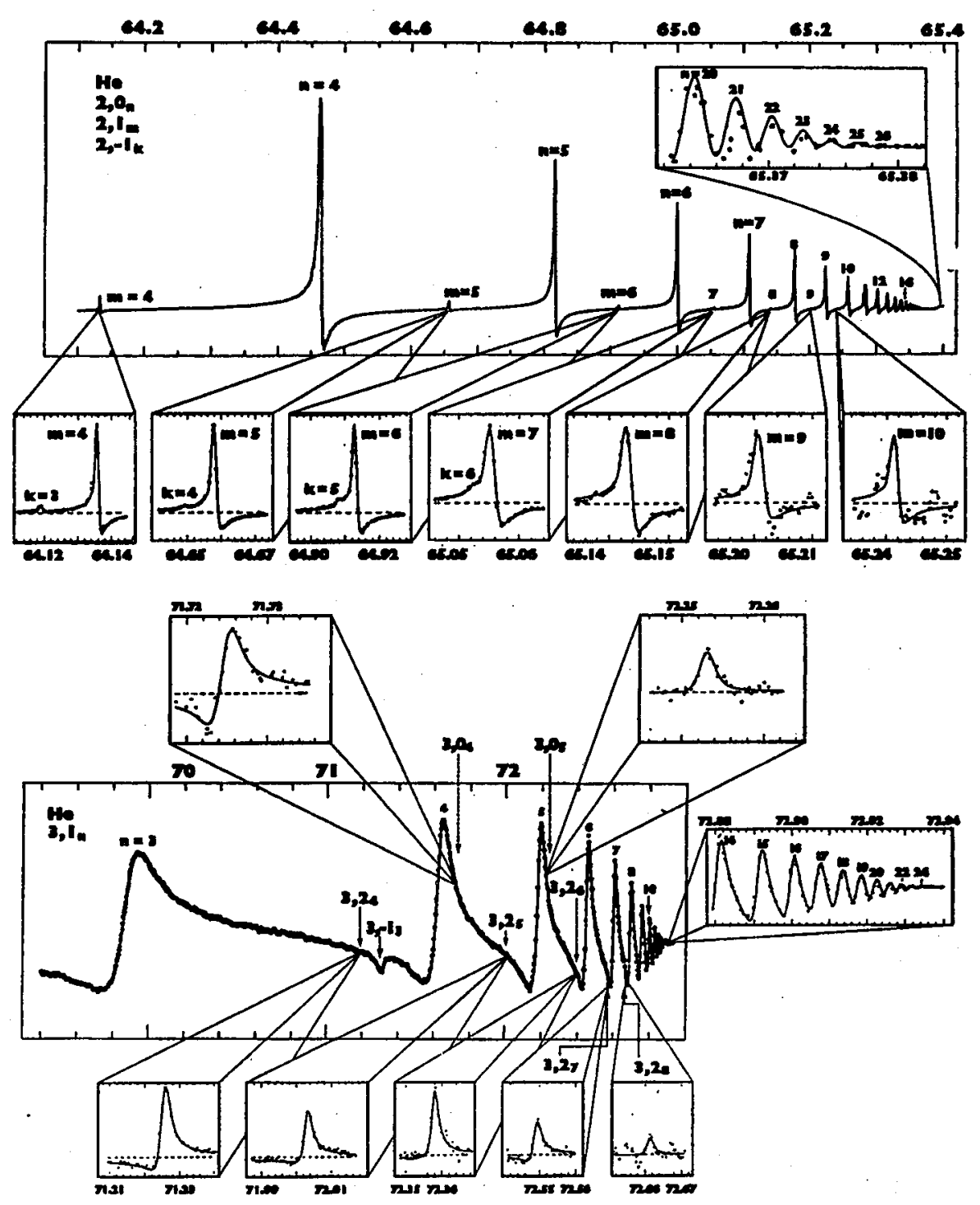

Fig. 1. Photoionization yield in helium: $N=2$ series (above), and $N=3$ series (below). Many resonances are observed for the first time [2]. 
energy levels and spectrum of the doubly-excited states of the helium atom. Doubly-excited autoionizing states of helium were first studied in the 1960's [3]. There was little progress thereafter until the early 1990 's when high-resolution beam lines at synchrotron light sources were introduced [4]. A major advance in resolution was achieved on Beamline 9.0.1 at the ALS, where a resolving power of 64000 has been demonstrated by measurement of the very narrow $2 p, 3 d\left(2,-1_{3}\right)$ state of helium [2].

Photoionization yield spectra for series converging to the $N=2$ and $N=3$ ionization thresholds are shown in Fig. 1. The notation intuitively means that the inner electron is excited to the $N=2$ or $N=3$ level, while the complete notation indicates the state of both electrons (both the old notation and the notation in hyperspherical coordinates are shown). These spectra show in remarkable detail the complexity of the spectrum of doubly-excited autoionizing states of helium.

\section{Argon}

An example of very high spectral resolution in the photoionization yield of argon [5] is shown in Fig. 2. Narrow window resonances $(3 s \rightarrow n p)$ and a resolution of $0.7 \mathrm{eV}$ is demonstrated.

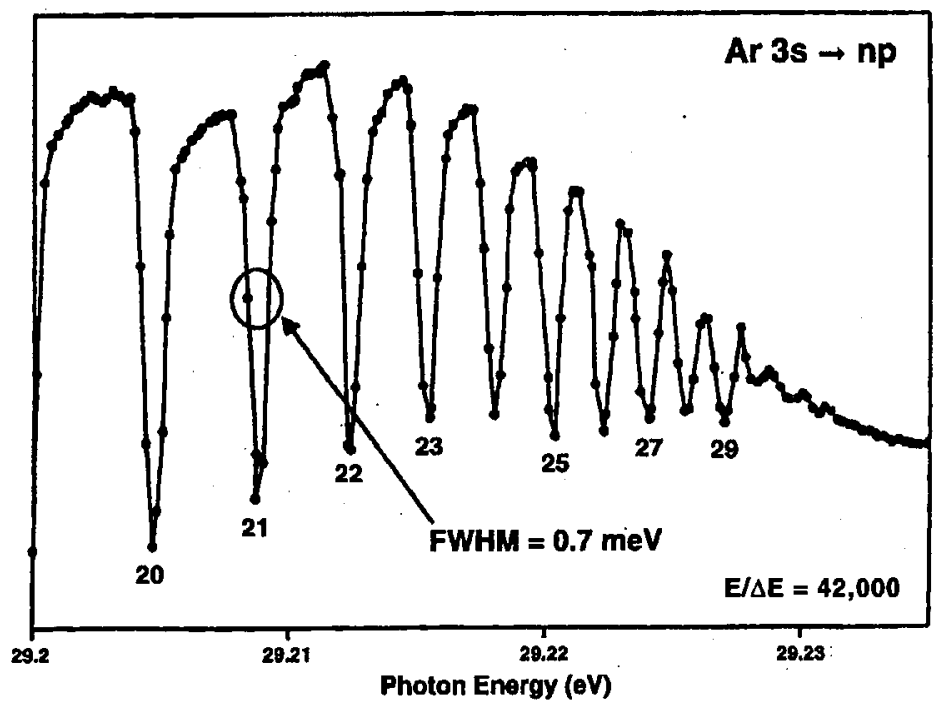

Fig. 2. Photoionization yield of argon showing high spectral resolution [5].

\section{Hollow lithium}

Lithium is the prototypical three-electron (four-body) atomic system. Hollow states are those with an empty inner shell, e.g., the $K$ shell, thus triply-excited states (all three electrons are excited) correspond to hollow states of lithium. Hollow lithium atoms can be produced by the impact of a single photon through 


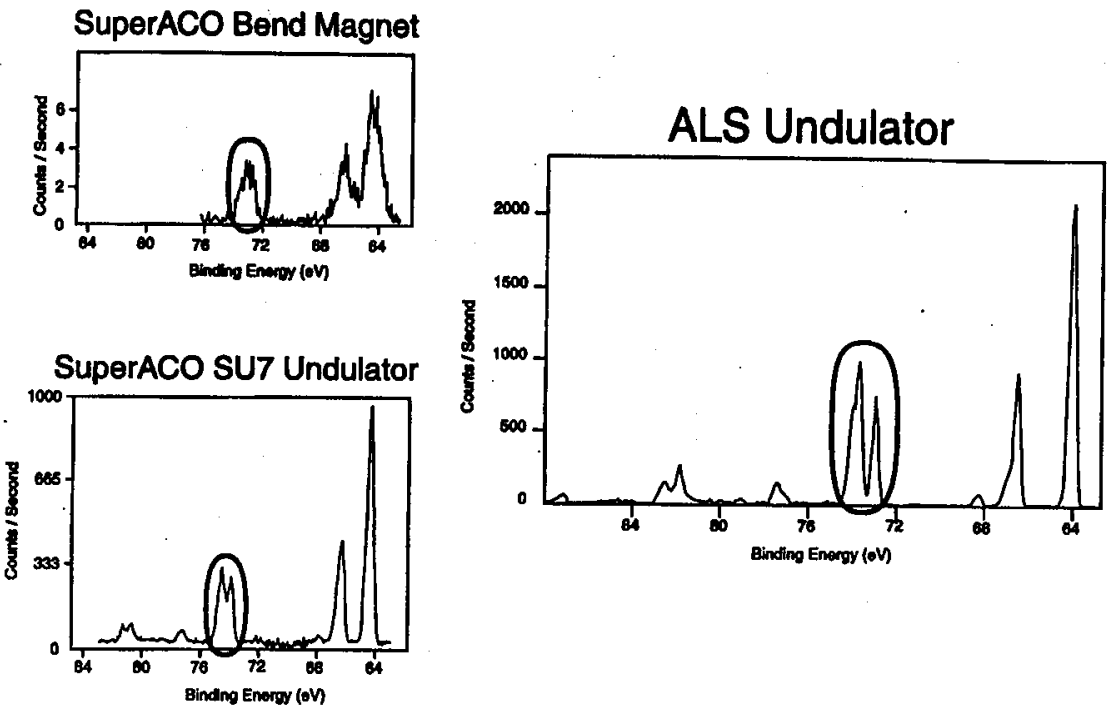

Fig. 3. Photoelectron spectra for emission from a hollow lithium state. Data from $\mathrm{Su}$ perACO bend magnet and undulator, and from ALS undulator showing the improved resolution on ALS Beamline 9.0.1. Photon and electron energies widths are reduced for the ALS measurement due to the high flux and spectral resolution on Beamline 9.0.1. (SuperACO bend magnet spectrum from F.J. Wuilleumier, private communication; SuperACO SU7 undulator spectrum from [6]; ALS undulator spectrum from [7]).

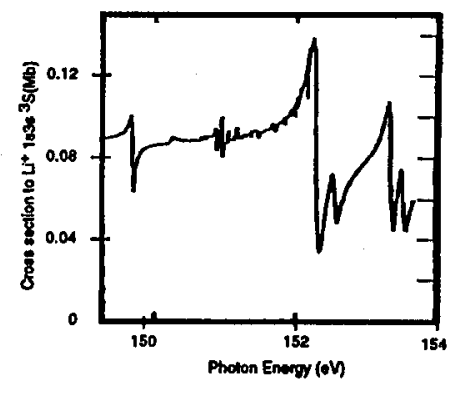

Theory

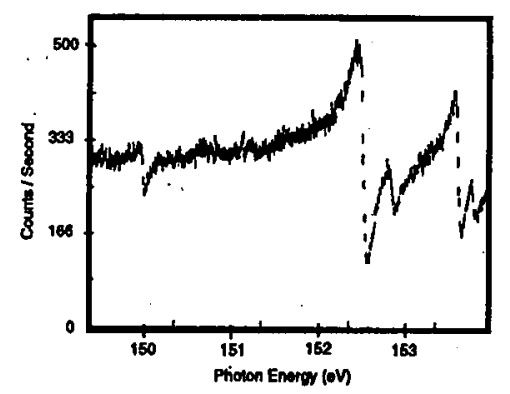

Experiment

Fig. 4. Hollow lithium: photoelectron emission to $\mathrm{Li}^{+} 1 s 2 s{ }^{3} S$ state, showing excellent agreement of theory and experiment [7].

electron correlation, and many resonant states have been studied by photoabsorption, by ion spectroscopy, and by photoelectron spectroscopy.

Hollow lithium has been studied by photoelectron spectroscopy at SuperACO (a second-generation light source in Orsay, France) on both a bend-magnet and an undulator beam line [6], as well as at the ALS [7]. A comparison of the 
electron energy resolution for the same states is shown in Fig. 3. Improvements in resolution, signal-to-noise ratio, and counting rate are evident.

Sample results of hollow lithium studies conducted on ALS Beamline 9.0.1 are shown in Fig. 4 [8]. Both the formation of the resonant states and the individual decay channels to different states of the $\mathrm{Li}^{+}$ion resulting from autoionization of the triply-excited states can be observed. Agreement between theory and experiment is excellent, with the measurements essentially confirming the theoretical calculations while highlighting a slight energy shift and a difference in cross section.

\section{Resonant Raman Auger in xenon}

The resonant Raman Auger effect circumvents the resolution limits imposed by lifetime broadening in Auger spectra $[8,9]$. The width of the resonant Raman Auger lines reflects only the instrumental contributions, i.e., combined widths of the photon and electron spectrometers. An example of the effect in xenon, measured with $65.11 \mathrm{eV}$ photons on Beamline 9.0.1 at the ALS [10], is shown in Fig. 5. This figure shows $4 d_{5 / 2}^{-1} 6 p$ to $5 p^{-2} 6 p$ transitions, and demonstrates the intensity distribution of spectator Auger transitions (the Auger electron is not from the same level as the excited electron). The line widths are $10 \mathrm{meV}$.
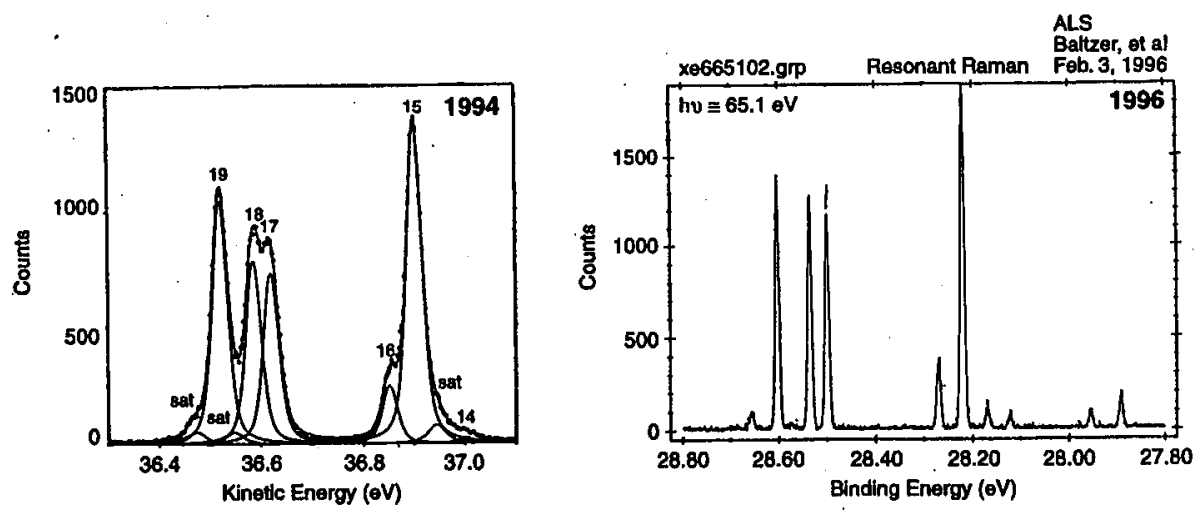

Fig. 5. Photoemission from xenon showing the $4 d^{-1} 6 p \rightarrow 5 p^{-2} 6 p$ Auger resonant Raman transition. (Spectrum from [9], ALS spectrum obtained using ALS Beamline 9.0.1 and Peter Baltzer's electron spectrometer; measurement by P. Baltzer and J.D. Bozek - private communication).

\section{Phosphorus trifluoride}

Ionization of an inner-shell electron in a molecule often leaves the system in a non-equilibrium nuclear geometry, and the electron-energy spectrum can show several kinds of splittings. Ionization of a $2 p$ electron in the phosphorus atom at the center of $\mathrm{PF}_{3}$ demonstrates three splittings: spin-orbit splitting $\left(2 p_{1 / 2}\right.$ and $2 p_{3 / 2}$ ), vibrational splitting, and ligand-field splitting, as shown in Fig. 6. 


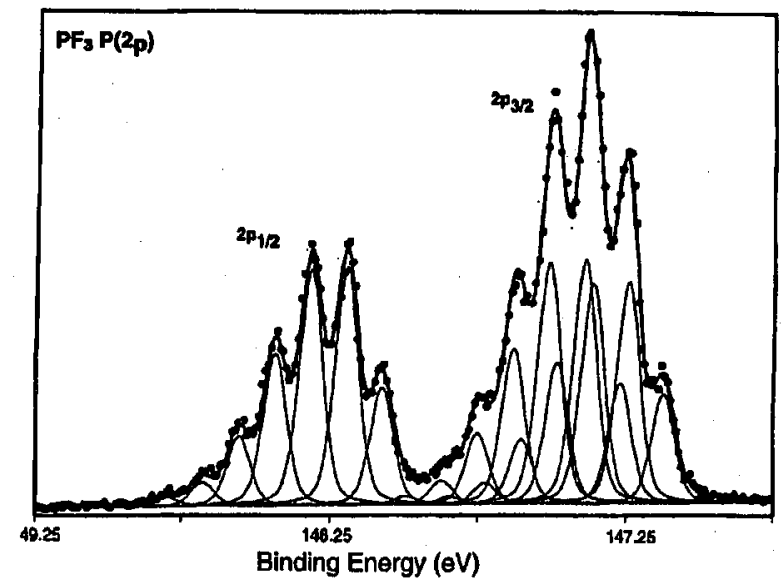

Fig. 6. Photoemission from $\mathrm{PF}_{3}$ showing vibrational and ligand-field splitting (J. Bozek, F. Schlachter, M. Lubell, T. Morgan, and C. Cisneros, 1995, unpublished).

\section{Conclusion}

Third-generation synchrotron-light sources provide new opportunities for research in many areas, including atomic and molecular physics. Ultra-high resolution photoionization and electron-emission spectroscopy applied to the study of atomic and molecular systems provide very sensitive tests of multibody theory.

\section{References}

[1] P. Heimann, D. Mossessian, J. Bozek, Advanced Light Source Note LSBL-239 (1995).

[2] K. Schulz, G. Kaindl, M. Domke, J.D. Bozek, P.A. Heimann, A.S. Schlachter, J.M. Rost, Phys. Rev. Lett. 77, 3086 (1996).

[3] R.P. Madden, K. Coddling, Phys. Rev. Lett. 10, 516 (1963).

[4] M. Domke, G. Remmers, G. Kaindl, Phys. Rev. Lett. 69, 1171 (1992).

[5] J.D. Bozek, private communication (1996).

[6] L. Journel, D. Cubaynes, J.-M. Bizau, S. Al Moussalami, B. Rouvellou, F.J. Wuilleumier, L. VoKy, P. Faucher, A. Hibbert, Phys. Rev. Lett. 76, 30 (1996).

[7] S. Diehl, D. Cubaynes, J.-M. Bizau, L. Journel, B. Rouvellou, S. Al Moussalami, F.J. Wuilleumier, E. Kennedy, N. Berrah, C. Blancard, T. Morgan, J. Bozek, A.S. Schlachter, L. Voky, P. Faucher, A. Hibbert, Phys. Rev. Lett. 76, 3915 (1996).

[8] B. Langer, N. Berrah, A. Farhat, O. Hemmers, J.D. Bozek, Phys. Rev. A 53, R1946 (1996).

[9] H. Akesela, S. Akesela, O.-P. Sairanen, A. Kivimaki, A. Naves de Brito, E. Nommiste, J. Tulkki, S. Svensson, A. Ausmees, S.J. Osborne, Phys. Rev. A 49, R4269 (1994).

[10] P. Baltzer, J.D. Bozek, private communication (1996). 